\title{
BIBLIOTECAS UNIVERSITARIAS ESPAÑOLAS EN LA WEB SOCIAL
}

\section{Pilar Grande-González y Pablo De-la-Fuente-Redondo}
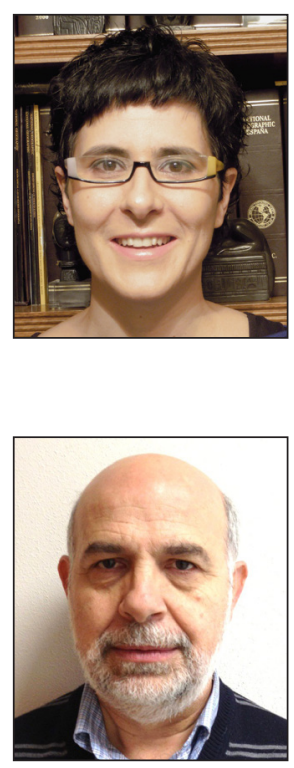

Pilar Grande-González, ingeniera informática por la Universidad de Valladolid (UVA), es profesora titular de escuela universitaria del Departamento de Informática de la UVA en la Escuela Universitaria de Informática del Campus de Segovia. Sus líneas de investigación se centran en la web social y las bibliotecas universitarias. Colabora con el Grupo de Investigación sobre Recuperación de Información y Bibliotecas Digitales (Grinbd) de la UVA.

http://orcid.org/0000-0002-0162-2827

Univ. de Valladolid, E.U. Informática. Campus de Segovia PI. Santa Eulalia, 9-11. 40005 Segovia, España pgrande@infor.uva.es

Pablo De-la-Fuente-Redondo es licenciado en físicas y doctor en ciencias por la Universidad de VaIladolid (UVA). Profesor titular de universidad del Departamento de Informática de la UVA, desempeña su labor profesional en la Escuela Técnica Superior de Ingeniería Informática del Campus de Valladolid. Sus líneas de investigación son bibliotecas digitales, interfaces de usuario, comprensión de texto y búsqueda en la web para dispositivos móviles. Desde su creación coordina el Grupo de Investigación sobre Recuperación de Información y Bibliotecas Digitales (Grinbd) de la UVA. http://orcid.org/0000-0002-4079-6467

Univ. de Valladolid, E.T.S. Ingeniería Informática. Campus Miguel Delibes, 47011 Valladolid, España pfuente@infor.uva.es

\section{Resumen}

Visión general de la aplicación de la web social en las bibliotecas universitarias españolas. Se han determinado, entre otras cuestiones, cuáles son los motivos por los que estas bibliotecas usan o no la web social, cuáles son los servicios 2.0 más y menos usados, con qué fines se utilizan estos servicios, los obstáculos a que se enfrentan las bibliotecas a la hora de llevar a cabo un proceso de este tipo, etc. Además, se ha tratado de cuantificar el grado de adopción de estas tecnologías con ayuda de nuevas medidas diseñadas en el proceso de investigación.

\section{Palabras clave}

Web social, Web 2.0, Tecnologías de comunicación, Bibliotecas universitarias, Bibliotecas académicas.

\section{Title: Spanish university libraries in the social web}

\section{Abstract}

Overview of the implementation of social web technologies in Spanish university libraries. The research made it possible to determine, among other issues, the reasons why these libraries use (or not) the social web, which 2.0 services are used more and less and for what purposes, obstacles faced by the libraries in carrying out a process of this type, etc. Furthermore, the study attempted to quantify the degree of adoption of these technologies with the help of new measures designed as part of the research process.

\section{Keywords}

Social web, Web 2.0, Communication technology, University libraries, Spanish university libraries, Academic libraries.

Grande-González, Pilar; De-la-Fuente-Redondo, Pablo. "Bibliotecas universitarias españolas en la web social". El profesional de la información, 2012, noviembre-diciembre, v. 21, n. 6, pp. 577-584.

http://dx.doi.org/10.3145/epi.2012.nov.04 


\section{Introducción}

La web social ha supuesto una revolución no sólo en la Web sino también en nuestra vida cotidiana. Cualquier internauta tiene a su disposición aplicaciones que le permiten representar un papel proactivo en internet sin necesidad de tener conocimientos tecnológicos avanzados. Los usuarios colaboran activamente entre ellos para beneficiarse de la "inteligencia colectiva", empresas de todo tipo tienen presencia en la Web, y se tiene la sensación de que quien no está en la Red no existe.

Las bibliotecas universitarias (en adelante, BU) no pueden permanecer ajenas y desde hace unos años vienen realizando esfuerzos para incorporarse de forma adecuada a esta corriente con el objetivo fundamental de acercarse a sus usuarios. En la actualidad un buen número de BU han adoptado la filosofía de la web social o se encuentran inmersas en plena fase de implementación.

\subsection{Antecedentes}

Existe abundante bibliografía sobre uso de aplicaciones de la web social en BU de todo el mundo. Por ejemplo, Shoniwa; Hall, 2007; Linh, 2008; Serrat-Brustenga; Sunyer-Lázaro, 2008; Xu et al., 2009; Aziz et al., 2011; Chan, 2000; Chua; Goh, 2010; Ebsco, 2010; García-Rivadulla, 2010; Hanz, 2010; Kim; Abbas, 2010; Tripathi; Kumar, 2010; Konsta, 2011; Li; Ruoyao; Bijuan, 2011; o Mahmood; Richardson, 2011. El análisis de la bibliografía indica que los servicios más utilizados son rss y blogs y los que menos marcadores sociales y podcasts, y que en los primeros puestos de uso no aparecen las redes sociales si bien existe una clara tendencia a su aumento; aunque cabe destacar la gran diversidad de resultados dependiendo de la fecha y el lugar de los estudios.

No se trata de tener muchos servicios de la web social sino de que sean rentables para la institución, que se puedan medir y tengan objetivos alcanzables

\section{Diseño de la investigación}

\subsection{Encuesta}

El objetivo fue averiguar el estado de aplicación de la web social en las BU españolas. Para conseguir una visión general del panorama nacional, los resultados de la encuesta debían dar respuesta a las preguntas:

- ¿Utilizan las BU tecnologías de la web social?

- ¿En qué fecha comenzaron su uso?

- ¿Existe una política de uso de la web social en las universidades/BU españolas?

- ¿Cómo se puede cuantificar el grado de adopción de las tecnologías de la web social que han llevado a cabo estas bibliotecas?

- ¿Cuáles son los servicios 2.0 más y menos usados?

- ¿Con qué fines se utilizan estos servicios?

\begin{tabular}{|l|c|c|}
\hline & BU & $\%$ \\
\hline Falta de capacitación del personal & 1 & 50 \\
\hline Política de la biblioteca & 1 & 50 \\
\hline Falta de personal & 1 & 50 \\
\hline Falta de tiempo & 1 & 50 \\
\hline $\begin{array}{l}\text { Dificultad al definir el modelo de presencia } \\
\text { en la Web de la biblioteca }\end{array}$ & 2 & 100 \\
\hline Otros & 0 & 0 \\
\hline
\end{tabular}

Tabla 1. Motivos por los que las BU no usan la web social (no total de bibliotecas que no utilizan la web social $=2$ )

- ¿Cuáles son los principales obstáculos a la hora de implementarlos?

- ¿Son reticentes las BU españolas a la introducción de nuevos servicios 2.0 ?

- ¿Llevan a cabo un registro de su actividad en la Web y en la web social en particular?

La encuesta constó de 70 preguntas organizadas en apartados de uso de: web 2.0, servicios 2.0, rss, blogs, podcasts, videocasts, mensajería instantánea, wikis, redes sociales, mundos virtuales, escritorios de trabajo online, multimedia compartido, navegación social, opac 2.0, y registro de datos. El número máximo de respuestas afirmativas posibles era de 227.

\subsection{Selección de bibliotecas}

Se invitó a participar a bibliotecarios de las universidades españolas y se intentó contactar con profesionales con un perfil 2.0 al objeto de obtener respuestas más fiables. Finalmente se recibieron 29 encuestas procedentes de 21 universidades. Según datos del Ministerio de Educación y Ciencia en España existen 76 universidades (52 públicas, 17 privadas y 7 pertenecientes a la iglesia católica), por tanto se obtuvo una participación del 27,6\%.

\subsection{Proceso de registro de datos}

Dado que en los sitios web de las BU podrían existir aplicaciones internas que no fuesen accesibles por el público en general e incluso servicios 2.0 que permaneciesen ocultos para los usuarios no registrados -con lo que no se podrían detectar en un análisis por contenido-, se descartó la idea inicial de realizar una evaluación externa del sitio web de las bibliotecas consideradas, y se optó por utilizar el cuestionario como única vía de recogida de datos. A petición de los bibliotecarios, el envío y recepción de formularios se llevó a cabo a través de una cuenta de correo electrónico creada para tal fin. La recogida de encuestas tuvo lugar durante el mes de junio de 2011 a lo largo de cuatro semanas.

\section{Resultados y discusión}

A continuación se muestran los resultados obtenidos.

\subsection{Uso de tecnologías de la web social}

De las 29 bibliotecas que han participado en el estudio tan sólo dos (UEMC y USC) no utilizaban la web social (aunque la de la USC acababa de iniciar un proceso de cambio a este respecto). El motivo fundamental que apuntan es una difi- 


\begin{tabular}{|l|c|c|}
\hline & BU & $\%$ \\
\hline Mejora la comunicación con los usuarios (cercanía) & 27 & 100,0 \\
\hline Es la biblioteca quién se acerca a los usuarios & 24 & 88,9 \\
\hline Aumenta la visibilidad & 26 & 96,3 \\
\hline Hace que la biblioteca sea más amigable & 21 & 77,8 \\
\hline Proyecta hacia el exterior el trabajo de los bibliotecarios & 23 & 85,2 \\
\hline Gratuidad de los servicios 2.0 & 22 & 81,5 \\
\hline Agiliza los procesos (rapidez) & 11 & 40,7 \\
\hline Actualización continua & 22 & 81,5 \\
\hline Sencillez (no se necesita ayuda de informáticos) & 23 & 85,2 \\
\hline Se utilizan a petición de los usuarios & 2 & 7,4 \\
\hline Permite que la biblioteca abra las 24 horas & 14 & 51,9 \\
\hline Ahorra costos & 7 & 25,9 \\
\hline Ahorra tiempo & 7 & 25,9 \\
\hline $\begin{array}{l}\text { Aumenta el número de préstamos y consultas de los fondos de la } \\
\text { biblioteca }\end{array}$ & 6 & 22,2 \\
\hline Refuerza la atención a los usuarios & 19 & 70,4 \\
\hline Los usuarios se sienten parte del proyecto & 12 & 44,4 \\
\hline $\begin{array}{l}\text { Aporta información sobre no de visitas, puntos desde dónde se accede, } \\
\text { etc. }\end{array}$ & 15 & 55,6 \\
\hline $\begin{array}{l}\text { Ayuda a permanecer informados sobre lo que hacen otras bibliotecas } \\
\text { universitarias e instituciones de interés }\end{array}$ & 22 & 81,5 \\
\hline $\begin{array}{l}\text { Facilita que los bibliotecarios estén en contacto con otros profesionales } \\
\text { que comparten los mismos intereses }\end{array}$ & 21 & 77,8 \\
\hline Otros motivos & 4 & 14,8 \\
\hline
\end{tabular}

Tabla 2. Motivos de uso de la web social (no total de bibliotecas que sí utilizan la web social $=27$ )

\subsection{Cuantificación del grado de adopción de tecnologías de la web social}

La tabla 4 registra los servicios $2.0 \mathrm{im}$ plementados en las BU participantes. Las 27 bibliotecas que han adoptado los principios de la web social no lo han hecho en la misma medida. Para tratar de cuantificar esta situación, hemos definido tres indicadores:

- n: número de respuestas afirmativas a la encuesta de la BU considerada (en total se podía alcanzar un valor máximo de 227, tal como se indicó en el apartado 2.1.).

- i: índice de aplicación de la web social en una BU determinada.

$\mathrm{i}=\mathrm{n} / 227 \times 100$

Una vez concluido el registro de los datos, se calculó el índice de aplicación de cada una de las bibliotecas. Los resultados se resumen en la tabla 5:

- La biblioteca del Área de Física de la Universidad de Sevilla (US2) ha obtenido el mayor índice (43,6 puntos) seguida a corta distancia de la Biblioteca de E.T.S. de Ingenieros de Telecomunicación de la UPM

cultad al definir el modelo de presencia en la Web por desconocimiento (tabla 1). Las 27 bibliotecas restantes destacan que el uso de estas tecnologías mejora la comunicación con sus usuarios al tiempo que aumenta la visibilidad de la biblioteca y proyecta hacia el exterior el trabajo que realizan. El centro se acerca más a los usuarios al acudir donde éstos se encuentran y permite que los bibliotecarios estén informados del modo en que se trabaja en otras instituciones similares. La gratuidad de estos servicios, su actualización continua y su facilidad de uso favorece la adopción (tabla 2).

\subsection{Fecha de inicio del uso de la web social}

Tan sólo 1 biblioteca (1 de 27, 3,7\%) lo hizo en el año 2005 coincidiendo con el momento en que se definió formalmente el concepto web 2.0. Posteriormente, durante 2008 y 2009 se produjo un incremento notable con un total de 8 $(29,6 \%)$ y 9 incorporaciones $(33,3 \%)$ respectivamente. Cerca del 63\% (17 de 27) se incorporaron en el período comprendido entre estos dos años (gráfico 1 y tabla 3).

\subsection{Política de uso}

Todos los bibliotecarios encuestados consideran importante que las BU incorporen aplicaciones de la web social en su sitio web y afirman que su uso beneficia o beneficiaría a sus bibliotecas y usuarios, a pesar de que tan sólo en un $34,5 \%$ de los centros (10 de 29) existe una política de uso de la web social. Sin embargo, se ha observado que la tendencia está cambiando y en gran parte de estas bibliotecas se está realizando un esfuerzo significativo para tratar de elaborar un plan estratégico de actuación al respecto.
(UPM3) (41,9 puntos).

- En el lado opuesto encontramos la Biblioteca de la Universidad de Cantabria (Unican) que obtuvo el índice de aplicación más bajo con sólo 9,3 puntos.

El análisis de las BU cuyo índice está por debajo del promedio muestra una situación dispar: hay 2 BU que incorporan sólo dos servicios de la web social, 3 con 4 servicios, una con 5,2 con 6 y una con 7 . Esto se debe a que o bien adopta-

\begin{tabular}{|l|c|l|c|}
\hline Biblioteca & Fecha & Biblioteca & Fecha \\
\hline UAH & oct 2008 & UNEX & 2008 \\
\hline UB1 & ene 2007 & UNICAN & jul 2007 \\
\hline UB2 & abr 2007 & UPCT & feb 2008 \\
\hline UBU & 2009 & UPM1 & jun 2010 \\
\hline UC3M & ene 2009 & UPM2 & ago 2008 \\
\hline UCA & dic 2007 & UPV & nov 2005 \\
\hline UCM1 & may 2008 & URV & sep 2009 \\
\hline UCM2 & (no lo usa) & US1 & dic 2008 \\
\hline UEMC & ene 2009 & US2 & feb 2008 \\
\hline UHU1 & (no responde) & USC & feb 2008 \\
\hline UHU2 & ene 2009 & UVA1 & (no lo usa) \\
\hline ULPGC & jun 2009 & UVA2 & may 2009 \\
\hline UM & may 2010 & UVA3 2009 \\
\hline UMH & nov 2007 & & dic 2009 \\
\hline UNAV & & & \\
\hline
\end{tabular}

Tabla 3. Fecha de adopción de la web social 


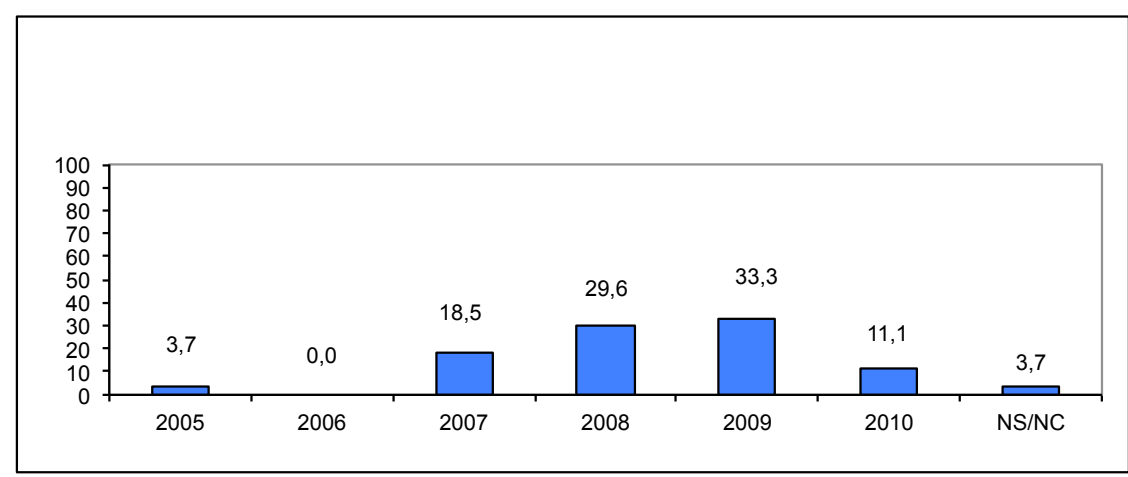

Gráfico 1. Año en que las BU empiezan a usar la web social (\%) trata de un dato erróneo, ya que si analizamos el contenido de los sitios web de estas bibliotecas encontramos mashups de forma más habitual a la detectada en la encuesta. Por tanto, parece que este registro erróneo se debe a un desconocimiento del término por parte de algunos de los bibliotecarios que respondieron a la encuesta. Le siguen los videocasts $(11,1 \%)$, podcasts $(14,8 \%)$ y wikis $(18,5 \%)$. Todos los servicios 2.0 considerados en la encuesta son usados por al menos una biblioteca universitaria española. ron pocos servicios o bien aplicaron las tecnologías 2.0 para usos simples como difundir noticias de la biblioteca, avisar de nuevos libros, sugerencias, etc. Por tanto, conseguir un alto índice de adaptación a la web social se debe a la forma en que se lleve a cabo la implementación de los servicios 2.0 en la biblioteca y no al número de tecnologías 2.0 que usan. Tal y como apuntan Torres-Salinas y Guallar (2009), "en última instancia cabría plantearse también si la web 2.0 no está trayendo una excesiva descentralización y un número elevado de canales de información para los profesionales de la información que impide que éstos puedan seguirlos y participar en todos con la intensidad que requieren".

\subsection{Casos especiales}

Dos BU obtuvieron un índice de aplicación de 0 puntos dado que no habían incorporado ninguno de los principios de la web social: la biblioteca de la Universidad Europea Miguel de Cervantes (UEMC) y la de la Facultad de Bioloxía de la Universidad de Santiago de Compostela (USC). Esta última sin embargo, al término del estudio acababa de incorporarse a la web social. Además los resultados obtenidos de los crais (centros de recursos para el aprendizaje y la investigación) de la Universitat de Barcelona y de la Universitat Rovira i Virgili han sido colocados en este apartado de casos especiales, al no tratarse de bibliotecas universitarias individuales propiamente dichas.

En cualquier caso hay que señalar que no se trata de tener muchos servicios de la web social sino de que sean rentables para la institución, y tengan objetivos alcanzables que se puedan medir. El índice de aplicación ayudó a cuantificar el grado de implantación, pero obviamente un valor elevado no debe identificarse con un mayor éxito ya que una biblioteca puede tener presencia en muchos medios 2.0 y tener un éxito menor que otra que está presente en menos pero de manera más cuidada y mejor gestionada.

\subsection{Servicios $\mathbf{2} .0$ más y menos usados}

Los de mayor aceptación (tabla 6) son los blogs y las redes sociales, ya que un $88,9 \%$ ( 24 de 27 ) de las BU que usan la web social los han incorporado a su sitio web, seguidos de cerca por el multimedia compartido (85,2\%), el rss $(81,5 \%)$, y la navegación social (63\%). En el lado contrario, los mundos virtuales son los menos utilizados $(3,7 \%)$ al ser adoptados por solamente una de estas BU. Este mismo porcentaje es obtenido por los mashups; sin embargo, en este caso se
Entre las redes sociales la gran triunfadora ha sido Facebook (gráfico 2), ya que el 95,8\% (23 de 24) tienen un perfil en esta plataforma. Este dato resulta lógico ya que, a 1 de enero de 2011 España tiene una población de 47,1 millones y 21,4 millones de usuarios de internet que se conectan a diario (INE, 2011). De ellos, 17,8 millones usa redes sociales, 16,3 millones son usuarios de Facebook y casi 3 millones de Twitter (Segovia, 2011).

Los podcasts y videocasts implementados muestran recorridos orientativos por la biblioteca, proporcionando información de carácter general o entrevistas a personajes de interés para la comunidad universitaria. Es una vía muy interesante que hace más atractivos a los usuarios los sitios web de las bibliotecas.

En las BU estudiadas existe un interés especial por mejorar la comunicación con sus usuarios, por lo que consideran importante adoptar estas tecnologías. Sin embargo, en numerosas ocasiones al usuario no se le dan las instrucciones suficientes sobre el uso de determinados servicios (mundos virtuales, escritorios de trabajo online, multimedia compartido, navegación social...), lo que va en detrimento de su uso. Este aspecto debería ser cuidado porque no supone una carga excesiva de trabajo y su mejora haría aumentar el uso. Por otro lado, algunos bibliotecarios lamentan la

\begin{tabular}{|c|c|c|}
\hline Biblioteca & $\mathrm{n}$ & i \\
\hline US2 & 99 & 43,6 \\
\hline UPM3 & 95 & 41,9 \\
\hline UM & 82 & 36,1 \\
\hline UCM1 & 80 & 35,2 \\
\hline ULPGC & 80 & 35,2 \\
\hline UNAV & 79 & 34,8 \\
\hline UC3M & 71 & 31,3 \\
\hline US1 & 67 & 29,5 \\
\hline UBU & 66 & 29,1 \\
\hline UHU1 & 65 & 28,6 \\
\hline UB2 & 63 & 27,8 \\
\hline UPM2 & 61 & 26,9 \\
\hline UCA & 58 & 25,6 \\
\hline UVA2 & 58 & 25,6 \\
\hline UPV & 54 & 23,8 \\
\hline UCM2 & 52 & 22,9 \\
\hline UNEX & 45 & 19,8 \\
\hline$U A H$ & 39 & 17,2 \\
\hline UPCT & 36 & 15,9 \\
\hline$U M H$ & 34 & 15,0 \\
\hline UPM1 & 27 & 11,9 \\
\hline UVA1 & 25 & 11,0 \\
\hline UHU2 & 24 & 10,6 \\
\hline UVA3 & 22 & 9,7 \\
\hline UNICAN & 21 & 9,3 \\
\hline \multicolumn{3}{|c|}{ Casos especiales } \\
\hline UEMC & 0 & 0 \\
\hline USC & 0 & 0 \\
\hline UB1 & 94 & 41,4 \\
\hline URV & 11 & 4,8 \\
\hline
\end{tabular}

Tabla 5. Valor del índice de aplicación de la web social en cada BU 


\begin{tabular}{|c|c|c|c|c|c|c|c|c|c|c|c|c|c|c|}
\hline & Rss & Blogs & Podcasts & Videocasts & $\begin{array}{l}\text { IM - } \\
\text { Chat }\end{array}$ & Wikis & $\begin{array}{c}\text { Redes } \\
\text { sociales } \\
\text { (SNS) }\end{array}$ & $\begin{array}{c}\text { Mundos } \\
\text { virtuales }\end{array}$ & $\begin{array}{c}\text { Escritorios } \\
\text { online }\end{array}$ & $\begin{array}{l}\text { Multimedia } \\
\text { compartido }\end{array}$ & $\begin{array}{c}\text { Navegación } \\
\text { social }\end{array}$ & $\begin{array}{c}\text { Opac } \\
2.0\end{array}$ & $\begin{array}{c}\text { Mashups } \\
\left({ }^{* *}\right)\end{array}$ & $\begin{array}{c}\text { Otros } \\
(*)\end{array}$ \\
\hline UAH & $x$ & $x$ & & & $x$ & $x$ & & & $x$ & $x$ & $x$ & & & \\
\hline UB1 & $x$ & $x$ & $x$ & & & $x$ & $x$ & & $x$ & $x$ & $x$ & & & \\
\hline UB2 & $x$ & $x$ & $x$ & & & & $x$ & & $x$ & $x$ & $x$ & $x$ & & \\
\hline UBU & $x$ & $x$ & & & $x$ & & $x$ & & $x$ & $x$ & & $x$ & & \\
\hline UC3M & & $x$ & & & & & $x$ & $x$ & $x$ & $x$ & & & & \\
\hline$U C A$ & $x$ & $x$ & & & $x$ & & $x$ & & & $x$ & & $x$ & & \\
\hline UCM1 & $x$ & $x$ & & $x$ & $x$ & & $x$ & & $x$ & $x$ & $x$ & $x$ & & \\
\hline UCM2 & $x$ & $x$ & & & $x$ & $x$ & $x$ & & $x$ & $x$ & $x$ & $x$ & & \\
\hline \multicolumn{15}{|l|}{ UEMC } \\
\hline UHU1 & $x$ & $x$ & & & $x$ & & $x$ & & $x$ & $x$ & $x$ & & & \\
\hline UHU2 & $x$ & & & & & & $x$ & & & & & & & \\
\hline ULPGC & $x$ & $x$ & & $x$ & $x$ & & $x$ & & $x$ & $x$ & $x$ & $x$ & & \\
\hline UM & $x$ & $x$ & & & $x$ & & $x$ & & & $x$ & $x$ & $x$ & & \\
\hline UMH & $x$ & $x$ & $x$ & & & & $x$ & & & & & & & \\
\hline UNAV & $x$ & $x$ & & & & & $x$ & & & $x$ & $x$ & $x$ & $x$ & $x$ \\
\hline UNEX & $x$ & $x$ & & & & & $x$ & & & $x$ & $x$ & $x$ & & \\
\hline UNICAN & & $x$ & & & & & $x$ & & & & & & & \\
\hline UPCT & $x$ & & & & $x$ & & $x$ & & & $x$ & & & & $x$ \\
\hline UPM1 & & $x$ & & & & & $x$ & & & $x$ & & $x$ & & \\
\hline UPM2 & $x$ & $x$ & & & $x$ & & $x$ & & $x$ & $x$ & $x$ & & & \\
\hline UPM3 & $x$ & $x$ & $x$ & $x$ & & & $x$ & & $x$ & $x$ & $x$ & & & \\
\hline UPV & $x$ & $x$ & & & $x$ & & $x$ & & $x$ & $x$ & & & & \\
\hline URV & & $x$ & & & & & & & & & & & & \\
\hline US1 & $x$ & $x$ & & & $x$ & $x$ & $x$ & & & $x$ & $x$ & & & \\
\hline US2 & $x$ & $x$ & & & $x$ & $x$ & $x$ & & $x$ & $x$ & $x$ & $x$ & & $x$ \\
\hline \multicolumn{15}{|l|}{ USC } \\
\hline UVA1 & $x$ & $x$ & & & & & & & & $x$ & $x$ & & & \\
\hline UVA2 & $x$ & $x$ & & & $x$ & & $x$ & & & $x$ & $x$ & $x$ & & \\
\hline UVA3 & & & & & $x$ & & $X$ & & $x$ & $x$ & $x$ & $x$ & & \\
\hline
\end{tabular}

Tabla 4. Servicios que ofrecen las BU encuestadas

$(* *)$ Mashups:

- Google books en el opac, Google maps en la Web, Google API feeds para la conexión web y blog de la biblioteca, Google calendar para las sesiones de formación (UNAV).

- En algunas bibliotecas también se usa Google maps para la geolocalización del edificio (p. e., UPM3) y de servicios.

$\left({ }^{*}\right)$ Otros:

- En la UNAV integran como boletín de novedades el agregador Netvibes con las RSS de su blog y las RSS generadas por el catálogo.

- En la UPCT utilizan SonoWeb en la página de inicio del Servicio de Documentación y en el Bibliotecario en línea; usan ShareThis para compartir la página de inicio de la web del Servicio de Documentación a través del correo, Facebook, etc.

- En la US2 disponen de una base de datos 2.0. Sobre Open Data Socrata han desarrollado Fistemática 2.0, una base de datos creada para mejorar el tiempo de proceso técnico y homogeneizar la asignación de materias y CDU, a la vez que se ofrece al usuario la búsqueda por cualquiera de los campos de la base de datos, orientándole sobre dónde están las obras de su interés si la biblioteca de libre acceso está ordenada por CDU, y facilitándole en un clic acceso a todos los documentos que tienen asignada una determinada materia.

http://opendata.socrata.com

http://opendata.socrata.com/Education/FISTEMATICA-2-0/3yqq-6ret

- Algunas BU utilizan Joomla o Drupal para crear repositorios o bibliotecas digitales.

poca participación de los lectores en redes sociales y wikis.

\subsection{Fines con que se usan los servicios 2.0}

Dado que el objetivo principal es mejorar la comunicación (tabla 2), las BU acuden a las plataformas donde se encuentran los usuarios para ofrecerles los servicios. Se centran en cuatro puntos:
- Ofrecer una imagen más cercana, por ejemplo, mostrando fotografías de los bibliotecarios, vídeos y fotografías de la biblioteca, etc. Esto hace que cuando el usuario utilice el servicio de chat no tenga esa sensación de impersonalidad que se produce de forma habitual en los servicios a distancia.

- Mantener informado al usuario: información general, 
noticias de la biblioteca, nuevas adquisiciones, libros, eventos que se van a llevar a cabo, recursos, servicio de referencia sobre el sitio web del centro, sobre las redes sociales... Las nuevas generaciones utilizan la Web de forma habitual como primera vía de consulta a la hora de buscar información y eso es algo que las BU deben tener muy presente.

Ayudar: recorridos orientativos por la biblioteca, consulta del catálogo, uso de bases de datos, marcadores sociales, etc.

Fomentar la participación: clubs de lectura, solicitar sugerencias y opiniones acerca de los servicios, invitar a actos, implementar foros de discusión sobre libros y registrar votaciones, facilitar la creación de folksonomías... En definitiva, hacer que el usuario se sienta partícipe de la comunidad.

Tal y como afirman Serrat y Sunyer (2008), "la biblioteca del siglo XXI no es un servicio estático sino que se configura como un servicio a la carta (just in time y just for me) y para ello cabe orientar todas las actuaciones hacia la máxima calidad, aplicando la tecnología y las nuevas herramientas digitales".

\subsection{Obstáculos para la implementación de estos ser- vicios}

En la mayoría de las BU (65,5\%, 19 de 29) el principal obstáculo es la ausencia de políticas claras al respecto. Si dispusieran de un documento marco que sirviese de guía para definir el modelo de presencia en la Web, los índices de participación en la web social serían mayores.

La adopción de servicios 2.0 es un proceso en pleno desarrollo, pero la falta de políticas claras dificulta su expansión

En ocasiones no existe en la página principal de la web de las bibliotecas un enlace a los servicios 2.0, lo que dificulta su localización por parte de los usuarios, que tal vez ni siquiera conocen su existencia.

Hay servicios como IM-chat (mensajería instantánea), blogs y redes sociales, que requieren una dedicación especial, por lo que a veces las bibliotecas optan por no implementarlos al no disponer de suficiente personal para atenderlos de la forma adecuada. Es evidente que si un blog no se mantiene actualizado pierde interés: requiere un esfuerzo diario por parte de los bibliotecarios para crear posts. Tener en funcionamiento IM-chat implica que un bibliotecario tenga que responder las cuestiones planteadas por el usuario de forma inmediata, durante un período de tiempo de entre tres y siete horas diarias (repartidas entre los distintos turnos que cubre el personal de la biblioteca). Y por último, no se trata sólo de estar presentes en las redes sociales, sino de participar de forma adecuada cuidando especialmente la imagen que se ofrece del centro.

Mención especial merece el opac 2.0 (opac social), ya que lo utiliza la mitad de las BU que usan la web social $(48,1 \%$,

\begin{tabular}{|l|r|r|}
\hline \multicolumn{1}{|c|}{ Servicio 2.0 } & BU & $\%$ \\
\hline Rss & 22 & 81,5 \\
\hline Blogs & 24 & 88,9 \\
\hline Podcasts & 4 & 14,8 \\
\hline Videocasts & 3 & 11,1 \\
\hline Mensajería instantánea & 15 & 55,6 \\
\hline Wikis & 5 & 18,5 \\
\hline Redes sociales & 24 & 88,9 \\
\hline Mundos virtuales & 1 & 3,7 \\
\hline Escritorio de trabajo online & 14 & 51,9 \\
\hline Multimedia compartido & 23 & 85,2 \\
\hline Navegación social & 17 & 63,0 \\
\hline Opac 2.0 & 13 & 48,1 \\
\hline Mashups & 1 & 3,7 \\
\hline Otros & 3 & 11,1 \\
\hline
\end{tabular}

Tabla 6. Servicios 2.0 que se utilizan en las BU españolas

13 de 27) (tabla 6). Según los bibliotecarios tienen fallos de pertinencia y exactitud de los resultados de las búsquedas respecto al opac tradicional, pero lo consideran un servicio de gran interés.

\subsection{Reticencias a la introducción de nuevos servicios 2.0}

Las BU que participaron en el estudio demostraron tener una "actitud 2.0". Incluso en los casos en que todavía no se han incorporado a la web social manifiestan su deseo de hacerlo. La mayoría es prudente y prefiere consolidar los servicios que ofrece antes de incorporar otros nuevos sin una planificación previa. Es de esperar que en un futuro próximo se produzca un gran incremento de la presencia de las BU españolas en la web social, determinado también por el simple hecho de que los usuarios ya están en ellas.

\subsection{Registro de su actividad en la Web}

$2 / 3$ de las $\mathrm{BU}(66,7 \%, 18$ de 27$)$ registran datos sobre su actividad en la Web, aunque de nuevo encontramos situaciones muy dispares: algunas llevan a cabo un registro exhaustivo, mientras que otras lo hacen en menor medida. La existencia de un plan de actuación sobre la presencia de la biblioteca en la web social determina un mayor registro de estos datos. Por otro lado, un $44,4 \%$ de las BU (12 de 27 ) utiliza aplicaciones gratuitas para registrar esta información. La mayor parte de ellas utiliza Google analytics (UBU, UCA, UHU2), otras utilizan las estadísticas que proporciona WordPress (UNEX), o bien diseñan sus propias hojas de cálculo para registrar esta información (UHU1). En la Universidad de Sevilla disponen de una wiki en la que sus bibliotecas registran sus datos trimestralmente (US1, US2). El éxito de Google analytics como herramienta de referencia en la analítica web se debe, además de su gratuidad, a que es intuitiva, flexible y potente.

Por último, en las BU estudiadas se detecta una clara preocupación por conocer datos sobre el uso e impacto de las herramientas 2.0 en la comunidad, sin embargo tan sólo un $25,9 \%$ (7 de 27 ) registran información al respecto. Ese mismo porcentaje de BU ha establecido indicadores para cuantificar cómo sus usuarios interactúan con ellas a través de la web social y algunas otras se encuentran en la actua- 
lidad en fase de diseño de los mismos. El análisis de estos indicadores les permitirá redefinir de forma adecuada su presencia en la web social.

\section{Conclusiones}

Se ha ofrecido una visión general de la adopción de la web social por parte de las BU, en junio de 2011. Debido a la situación de constante cambio, algunos datos registrados puedan quedar pronto desfasados. De hecho, una vez finalizado el período de investigación se han detectado cambios sustanciales en los sitios web de algunas de las bibliotecas que fueron analizadas, debido a que han implantado nuevos servicios o mejorado los existentes. Todas estas bibliotecas -y otras muchas que no han participado en el estudio- están realizando esfuerzos importantes para mejorar su presencia en la web social. Con este trabajo se pretende fomentar un aprendizaje entre las bibliotecas poniendo en común sus experiencias.

Sólo 2 de las 29 BU encuestadas no utilizan ninguna tecnología de la web social. Entre las restantes, 24 utilizan menos de 4 servicios. Los blogs y las redes sociales son los más utilizados $(88,9 \%)$, seguidos de cerca por el multimedia compartido $(85,2 \%)$ y el rss $(81,5 \%)$, mientras que los mundos virtuales (por ej. Second Life) son los menos utilizados $(3,7 \%)$. La adopción de servicios 2.0 en estas bibliotecas es un proceso en pleno desarrollo, pero la falta de políticas claras al respecto dificulta su expansión, aspecto que se intenta subsanar en la actualidad. Además, ciertos servicios (IM, redes

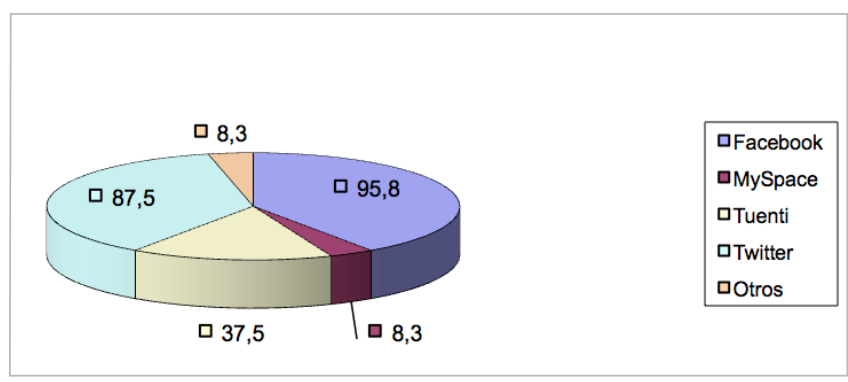

Gráfico 2. Presencia de las BU en redes sociales

sociales y blogs) requieren mayor dedicación por parte de los bibliotecarios por lo que en ocasiones no se implementan. Sin embargo, existe una corriente muy positiva en el mundo bibliotecario que apuesta decididamente por el concepto de biblioteca 2.0 lo que sin duda provocará grandes avances en su adopción en todo el territorio nacional.

La biblioteca universitaria debe tener presencia donde la tengan sus usuarios, pero no de forma experimental, sino planificada al detalle con anterioridad. Los departamentos de informática de las universidades y las BU deben trabajar conjuntamente para favorecer el salto a la web social, que debería producirse a corto plazo en todas las bibliotecas que todavía no lo hayan dado, y para ayudar a aquellas que ya están presentes en ella. Estas BU deben estar preparadas ante las tendencias actuales en comunicación para no quedar al margen de las próximas revoluciones que se originen en la Web.

\begin{tabular}{|c|c|c|}
\hline ID & Universidad & Biblioteca \\
\hline$U A H$ & Univ. de Alcalá de Henares & Biblioteca de la Univ. de Alcalá de Henares \\
\hline UB1 & Universitat de Barcelona & Centro de Recursos para el Aprendizaje y la Investigación (CRAI) \\
\hline UB2 & Universitat de Barcelona & Biblioteca del Campus de Ciències de la Salut de Bellvitge \\
\hline UBU & Univ. de Burgos & Biblioteca Universitaria \\
\hline$U C 3 M$ & Univ. Carlos III de Madrid & Biblioteca de la Univ. Carlos III \\
\hline UCA & Univ. de Cádiz & Biblioteca de Campus Río San Pedro \\
\hline UCM1 & Univ. Complutense de Madrid & Biblioteca de Ciencias Políticas y Sociología \\
\hline UCM2 & Univ. Complutense de Madrid & Biblioteca de Ciencias Económicas y Empresariales \\
\hline UEMC & Univ. Europea Miguel de Cervantes & Biblioteca Universitaria \\
\hline UHU1 & Univ. de Huelva & Biblioteca Universitaria \\
\hline UHU2 & Univ. de Huelva & Biblioteca de la Escuela Técnica Superior de Ingeniería \\
\hline ULPGC & Univ. de Las Palmas de Gran Canaria & Biblioteca de Educación Física \\
\hline UM & Univ. de Murcia & Biblioteca General \\
\hline$U M H$ & Univ. Miguel Hernández & Biblioteca de la Univ. Miguel Hernández \\
\hline UNAV & Univ. de Navarra & Biblioteca de la Univ. de Navarra \\
\hline UNEX & Univ. de Extremadura & Biblioteca Universitaria \\
\hline UNICAN & Univ. de Cantabria & Biblioteca Universitaria \\
\hline UPCT & Univ. Politécnica de Cartagena & Servicio de Documentación UPCT \\
\hline UPM1 & Univ. Politécnica de Madrid & $\begin{array}{l}\text { Biblioteca de la Escuela de Ingeniería Aeronáutica y del Espacio. EIAE (ETSI Aeronáuticos - EUIT } \\
\text { Aeronáutica) }\end{array}$ \\
\hline UPM2 & Univ. Politécnica de Madrid & Biblioteca de la Escuela Técnica Superior de Ingenieros Industriales \\
\hline UPM3 & Univ. Politécnica de Madrid & Biblioteca de la Escuela Técnica Superior de Ingenieros de Telecomunicación \\
\hline UPV & Univ. Politécnica de Valencia & Biblioteca del Campus de Alcoy \\
\hline URV & Universitat Rovira i Virgili & Centro de Recursos para el Aprendizaje y la Investigación (CRAI) \\
\hline US1 & Univ. de Sevilla & Biblioteca de Farmacia \\
\hline US2 & Univ. de Sevilla & Biblioteca del Área de Física \\
\hline USC & Univ. de Santiago de Compostela & Biblioteca de la Facultade de Bioloxía \\
\hline UVA1 & Univ. de Valladolid & Biblioteca de la Yutera \\
\hline UVA2 & Univ. de Valladolid & Biblioteca de la Facultad de Ciencias Económicas y Empresariales \\
\hline UVA3 & Univ. de Valladolid & Biblioteca General Universitaria "Reina Sofía" \\
\hline
\end{tabular}

Tabla 7. Relación de BU que han cumplimentado la encuesta 


\section{Agradecimientos}

Este informe no habría sido posible sin la inestimable colaboración de los bibliotecarios que ayudaron a difundir la encuesta e hicieron llegar sus observaciones y comentarios a través de los cuestionarios y en entrevistas y conversaciones telefónicas.

Mención especial merece la colaboración prestada por Nieves González-Fernández-Villavicencio (Universidad de SeviIla) que revisó la encuesta y el estudio realizado.

Este trabajo ha sido parcialmente financiado por el proyecto Pavicylex (VA025A10-2) del Programa General de Apoyo a Proyectos de Investigación de la Junta de Castilla y León.

Se trata de tener presencia donde la tengan sus usuarios, pero no de forma experimental, sino previamente planificada al detalle

\section{Referencias bibliográficas}

Aziz, Rafidah-Abdul; Arif, Zuraidah; Ramly, Ruzita; AlduIlah, Che-Zainab; Husaini, Haslinda. "The implications of library 2.0 tools in Malaysian Academic Library towards reference services". En: Asia Pacific conf. library \& information education \& practice, 2011, pp. 579-588.

http://eprints.uitm.edu.my/3625/1/SP_TIO11_62.pdf

Chan, Hong-Han-Rosita. "Murdoch University Library: a web 2.0 journey". En: ALSR 2010: Academic librarian 2: Singing in the rain, 2010.

http://www.lib.polyu.edu.hk/ALSR2010/programme/pdf/ Session10B_Chan.pdf

Chua, Alton Y. K.; Goh, Dion H. "A study of web 2.0 applications in library websites". Library and information science research, 2010, v. 32, n. 3, pp. 203-211.

http://dx.doi.org/10.1016/j.lisr.2010.01.002

Ebsco. "Social media in libraries (European survey findings)". En: IFLA 2010, 2010.

http://www.slideshare.net/jhoussiere/social-media-usagein-libraries-in-europe-survey-teaser

García-Rivadulla, Sandra. "Actitud 2.0: usos de la web social en las bibliotecas universitarias uruguayas", 2010.

http://eprints.rclis.org/handle/10760/13978

Gerolimos, Michalis; Konsta, Rania. "Services for academic libraries in the new era". D-lib magazine, 2011, Jul-Ag., v. 17, n. 7-8.

http://www.dlib.org/dlib/july11/gerolimos/07gerolimos. html

Han, Zhiping; Quan-Liu, Yan. "Web 2.0 applications in top Chinese university libraries". Library hi tech, 2010, v. 28, n. 1, pp. 41-62.

http://dx.doi.org/10.1108/07378831011026689

INE. "Encuesta sobre equipamiento y uso de tecnologías de información y comunicación en los hogares. Año 2011". No- tas de prensa, 5 octubre 2011.

http://www.ine.es/prensa/np678.pdf

Kim, Yong-Mi; Abbas, June. "Adoption of library 2.0 functionalities by academic libraries and users: a knowledge management perspective". Journal of academic librarianship, 2010, v. 36, n. 3, pp. 211-218.

http://dx.doi.org/10.1016/j.acalib.2010.03.003

Li, Si; Ruoyao, Shi; Bijuan, Chen. "An investigation and analysis of the application of web 2.0 in Chinese university libraries". The electronic library, 2011, v. 29, n. 5, pp. 651668.

http://dx.doi.org/10.1108/02640471111177080

Linh, Nguyen-Cuong. "A survey of the application of web 2.0 in Australasian university libraries". Library hi tech, 2008, v. 26, n. 4, pp. 630-653.

http://dx.doi.org/10.1108/07378830810920950

Mahmood, Khalid; Richardson, John V. "Adoption of web 2.0 in US academic libraries: a survey of ARL library websites". Program: electronic library and information systems, 2011, v. 45, n. 4, pp. 365-375

$h t t p: / / d x$. doi.org/10.1108/00330331111182085

Segovia, Adrián. “¿Quién aporta más audiencia: Google, Facebook o Twitter?" El país. Estrategia digital, 2011.

http://blogs.elpais.com/estrategia-digital/2011/03/googlefacebook-twitter.html

Serrat-Brustenga, Marta; Sunyer-Lázaro, Sílvia. "El centro de recursos para el aprendizaje y la investigación (crai) en permanente transformación: servicios y recursos para el nuevo usuario 2.0". En: X Jornadas de gestión de la información, 2008.

http://upcommons.upc.edu/e-prints/bitstream/2117/2427/1/ serrat_sedic_ponencia.pdf

Shoniwa, Pride; Hall, Hazel. "Library 2.0 and UK academic libraries: drivers and impacts". New review of information networking, 2007, v. 13, n. 2, pp. 69-79.

http://www.soc.napier.ac.uk/ hazelh/esis/shoniwa_hall_ manu.pdf

Torres-Salinas, Daniel; Guallar, Javier. "Evaluación de DocuMenea, sistema de promoción social de noticias de biblioteconomía y documentación". El profesional de la información, 2009, marzo-abril, v. 18, n. 2, pp. 171-179.

http://www.elprofesionaldelainformacion.com/contenidos/2009/ marzo/07.pdf

http://dx.doi.org/10.3145/epi.2009.mar.07

Tripathi, Manorama; Kumar, Sunil. "Use of web 2.0 tools in academic libraries: a reconnaissance of the international landscape". The international information and library review, 2010, v. 42, n. 3, pp. 195-207.

http://review-mobile.wikispaces.com/file/view/article.pdf http://dx.doi.org/10.1016/j.iilr.2010.07.005

Xu, Chen; Ouyang, F.; Chu, H. "The academic library meets web 2.0: applications and implications". The journal of academic librarianship, 2009, v. 35, n. 4, pp. 324-331. http://eprints.rclis.org/bitstream/10760/10779/1/The Academic_Library_Meets_Web_2.0-_Applications_\&_ Implications.pdf 